\title{
A Robust Bisection-Based Estimator for TOA-Based Target Localization in NLOS Environments
}

\author{
Slavisa Tomic, Marko Beko, Rui Dinis, and Paulo Montezuma
}

\begin{abstract}
This letter addresses the problem of target localization in harsh indoor environments based on range measurements. To mitigate the non-line-of-sight (NLOS) bias, we propose a novel robust estimator by transforming the localization problem into a generalized trust region sub-problem framework. Although still non-convex in general, this class of problems can be readily solved exactly by means of bisection procedure. The new approach does not require to make any assumptions about the statistics of NLOS bias, nor to try to distinguish which links are NLOS and which are not. Unlike the existing algorithms, the computational complexity of the proposed algorithm is linear in the number of reference nodes. Our simulation results corroborate the effectiveness of the new algorithm in terms of NLOS bias mitigation and show that the performance of our estimator is highly competitive with the performance of the stateof-the-art algorithms. In fact, they show that the novel estimator outperforms slightly the existing ones in general, and that it always provides a feasible solution.
\end{abstract}

Index Terms-Robust localization, time of arrival (TOA), non-line-of-sight (NLOS), generalized trust region sub-problem (GTRS), wireless sensor network (WSN).

\section{INTRODUCTION}

$\mathbf{T}$ ARGET localization in indoor environments has received immense attention recently in the research society, owing to its pertinence in both military and commercial applications [1], [2]. In harsh indoor environments, where most connections are non-line-of-sight (NLOS), a frequently opted solution is to rely on reference nodes (anchors) and (noisy) range measurements between the target and anchors. These are extracted from time of arrival (TOA), received signal strength or other characteristic of the radio signal [3]-[6]. However, in harsh indoor environments, the influence of NLOS bias might significantly degrade the localization performance. Hence, mitigation of NLOS bias is an important task in practice [7].

Various techniques for NLOS bias mitigation can be found in the literature [1]. Some of them are based on identifying the

Manuscript received July 11, 2017; accepted August 7, 2017. Date of publication August 11, 2017; date of current version November 9, 2017. This work was partially supported by Fundação para a Ciência e a Tecnologia under Project PEst-OE/EEI/UI0066/2014, Project UID/EEA/50008/2013, Project UID/EEA/50009/2013, and Program Investigador FCT under Grant IF/00325/2015. The associate editor coordinating the review of this letter and approving it for publication was Y. Shen. (Corresponding author: Slavisa Tomic.)

S. Tomic is with ISR/IST, LARSyS, Universidade de Lisboa, 1049-001 Lisbon, Portugal (e-mail: stomic@isr.ist.utl.pt).

M. Beko is with CICANT-CIC.DIGITAL, Universidade Lusófona de Humanidades e Tecnologias, 1749-024 Lisboa, Portugal, and also with CTS/UNINOVA, FCT/UNL, 2829-516 Caparica, Portugal (e-mail: beko.marko@ulusofona.pt).

R. Dinis is with the Instituto de Telecomunicações, 1049-001 Lisboa, Portugal (e-mail: rdinis@fct.unl.pt).

P. Montezuma is with the Department of Electrical Engineering, FCT/UNL, 2829-516 Caparica, Portugal (e-mail: pmc@uninova.pt).

Digital Object Identifier 10.1109/LCOMM.2017.2737985
NLOS links [8], [9], which are then either discarded or used to improve the performance, while others are robust estimators [10], [11] which are generally more resilient to NLOS bias. A robust estimator based on semidefinite programming (SDP) relaxation technique was introduced in [10] to cope with the NLOS bias. Even though this estimator performs well in adverse environments, its computational complexity grows rapidly with the network size. Another SDP estimator was presented in [7]. This estimator tries to mitigate the NLOS bias by properly handling the NLOS links, but disregards a relationship established between optimization variables and is $\gamma$-dependent, where $\gamma$ is a penalization term that prevents the problem to be ill-posed. In [11], a robust second-order cone programming (SOCP) estimator was described. It provides a relatively fast and accurate solution, but it is not always feasible. A perturbed edge-based SDP (PESDP) estimator was proposed in [12]. This estimator was originally designed for cooperative localization, and relies on perturbation terms introduced in target/target links to enhance its performance.

All of the above algorithms for TOA-based target localization in NLOS environments employ sophisticated mathematical tools which raise severely their computational complexity, and thus, their execution time. In huge contrast to them, here, we propose a novel robust estimator whose solution is exactly obtained by nothing other than a bisection procedure. The proposed algorithm requires to know the upper bound on the magnitude of NLOS bias, which is relatively easy to estimate in practice during the training phase [10], [11], when the true locations of sensors are available. It does not require to distinguish between line-of-sight (LOS) and NLOS links, nor to know the statistics of the NLOS bias. We take the NLOS bias as a nuisance parameter, and develop a robust estimator that mitigates its influence. By applying robust squared-range (RSR) and weighted least squares (WLS) criterion, we convert the originally non-convex problem into a generalized trust region sub-problems (GTRS) framework, whose solution is readily obtained by a bisection procedure [13]. Unlike the described algorithms, the computational complexity of the new one is linear in the number of anchors, and its performance is highly competitive with the state-ofthe-art algorithms.

\section{Problem Formulation}

Let $\boldsymbol{x}, \boldsymbol{a}_{i} \in \mathbb{R}^{k}$ represent respectively the unknown location of the target and the known location of the $i$-th anchor, $i=1, \ldots, N$, in a $k$-dimensional wireless sensor network ( $k=2$ or 3 ). The range measurements between the target and the $i$-th anchor [7], [10]-[12], [14] are modeled as

$$
d_{i}=\left\|\boldsymbol{x}-\boldsymbol{a}_{i}\right\|+b_{i}+n_{i}, \quad \text { for } i=1, \ldots, N,
$$

where $n_{i}$ is the measurement noise assumed to follow a zero-mean Gaussian distribution with variance $\sigma_{i}^{2}$, 